\title{
What is learned in spatial contextual cuing- configuration or individual locations?
}

\author{
YUHONG JIANG and LAURA C. WAGNER \\ Massachusetts Institute of Technology, Cambridge, Massachusetts
}

\begin{abstract}
With the use of spatial contextual cuing, we tested whether subjects learned to associate target locations with overall configurations of distractors or with individual locations of distractors. In Experiment 1 , subjects were trained on 36 visual search displays that contained 36 sets of distractor locations and 18 target locations. Each target location was paired with two sets of distractor locations on separate trials. After training, the subjects showed perfect transfer to recombined displays, which were created by combining half of one trained distractor set with half of another trained distractor set. This result suggests that individual distractor locations were sufficient to cue the target location. In Experiment 2, the subjects showed good transfer from trained displays to rescaled, displaced, and perceptually regrouped displays, suggesting that the relative locations among items were also learned. Thus, both individual target-distractor associations and configural associations are learned in contextual cuing.
\end{abstract}

Humans have severe limitations in cognitive processing. We sometimes fail to see objects that are right in front of us (Levin \& Simons, 1997; Mack \& Rock, 1998; Rensink, O’Regan, \& Clark, 1997; Shore \& Klein, 2000), we rely on written, laid-out instructions to keep track of what we are supposed to be doing (Ballard, Hayhoe, \& Pelz, 1995), and we cannot maintain a conversation while turning left in traffic (Pashler, 1994). Yet in many aspects of everyday information processing we are virtuosos: We can recognize an object, a face, or a scene in one fifth of a second (Liu, Harris, \& Kanwisher, 2002; Potter, 1976; Thorpe, Fixe, \& Marlot, 1996), type 70 words per minute (Keele \& Hawkins, 1982), and redirect our gaze to the most important information in the visual field several times per second (Irwin, 1992). Efficient visual processing is achieved because, on the one hand, powerful mechanisms of selective attention are used to filter out task-irrelevant information (Pashler, 1998; Yantis, 1998), and on the other hand, the outside world is frequently relied upon as the veridical "memory" to minimize the reliance on the internal, short-term memory representation (Ballard et al., 1995; Hayhoe, 2000; O’Regan, 1992).

Still, a lot of visual information is extracted at any given moment, even though not all of it reaches awareness. Implicit learning allows the visual system to quickly extract stimulus regularities (Reber, 1989), such as the repetition of a target's color or location (Maljkovic \& Nakayama, 1994, 1996), a repeated sequence of target locations (Nissen \& Bullemer, 1987), and the set of other objects within

The present study is supported by a Helen Hay Whitney Research Fellowship. We thank Molly Potter and Marvin Chun for discussions and John Seamon, Kyungmouk Lee, Jennifer Stolz, and two anonymous reviewers for their comments. Correspondence should be sent to Y. Jiang, 33 Kirkland Street, Room 820, Department of Psychology, Harvard University, Cambridge, MA 02138 (e-mail: yuhong@mit.edu). which the target is presented (Chun \& Jiang, 1999). In contrast to the small capacity of visual attention or shortterm memory, implicit visual learning can process hundreds of complex visual displays to "detect" repetitions. It facilitates processing of repeated displays after just four or five exposures (Chun, 2000) and can last for at least a week (Chun \& Jiang, 2003). Implicit visual learning thus affords us with a powerful mechanism to represent the enormously rich and complex, yet frequently redundant, visual environment. This study addresses what information is learned in visual search in a spatial contextual cuing paradigm (Chun \& Jiang, 1998). In the following paragraphs, we shall first provide an overview of contextual cuing and then test which kind of information - the pattern formed by the items or each individual location - is learned in contextual cuing.

In a series of studies, Chun and colleagues (Chun, 2000; Chun \& Jiang, 1998, 1999, 2003; Jiang \& Chun, 2001; Olson \& Chun, 2001, 2002) have demonstrated that invariant information, extracted from past experience, significantly enhances visual search even when observers are unaware of the learning and memory. The effect of implicit learning on attentional deployment is called contextual cuing. The subjects in these studies are typically tested in visual search tasks that require slow scrutiny of the displays, such as looking for a T among Ls. They perform the search task in two dozen blocks, each block containing 24 different search displays. Although the subjects are unaware of it, half of the search displays are repeated across blocks, so the subjects will have searched from the same displays two dozen times in the experiment. These old displays are intermixed with new displays containing new distractor locations. Over a 1-h training session, search speed improves steadily for both new and old displays, but response times (RTs) for the old displays gradually diverge from and becomes faster than RTs for the new displays. The RT 
advantage reflects learning of the repeated context. Learning and memory in this task are implicit. The subjects rarely report noticing the repetitions. They cannot recognize old displays (Chun \& Jiang, 1998), nor can they point out the target location within old displays (Chun \& Jiang, 2003).

What is learned in spatial contextual cuing? Past studies have shown that it is not target locations, because these are repeated in both new and old displays. Nor is it the target identity or its associated manual responses, because the target shape and orientation differ randomly on every trial. Perceptual familiarity with a certain set of distractor locations is also insufficient, because no learning is observed if the repeated distractor set does not predict the target location (Chun \& Jiang, 1998; see also Wolfe, Klempen, \& Dahlen, 2000). Thus, to search more quickly from the old displays, subjects have to learn the context created by the repeated distractor locations and rely on the consistent association between distractor locations and the target location to facilitate visual search. But what do subjects learn from the repeated distractor locations? Do they extract the pattern formed by all items (configuration) or do they simply learn where each individual item is?

Chun and Jiang (1998) argued that the global spatial layout formed by all items is extracted, which becomes an effective cue for the target location. This account is plausible, because the visual system represents the configuration of a search display obligatorily in perception (Yantis, 1992) and short-term memory (Jiang, Olson, \& Chun, 2000). Subjects typically extract the imaginary geometric configuration formed by all elements. However, because both the configuration formed by the locations and the individual locations themselves have been repeated in past studies of contextual cuing, we do not know which information drives the effect. Learning could be driven by associating the target location with each distractor location. For instance, subjects may (implicitly) remember that if a distractor is at the leftmost corner, then the target is $2^{\circ}$ above fixation.

In a study in which the authors compared distractors adjacent to the target with distractors farther away from the target, Olson and Chun (2002) observed significant contextual cuing only for the adjacent, local context. They further showed that the lack of a context effect from the far distractors was not because the geometric distance was too far, but because the variable, adjacent distractors interfered with the learning of the far context. If the adjacent distractors were removed, a consistent far context was sufficient to produce contextual cuing. Nonetheless, the fact that adjacent and far distractors had different effects suggests that learning was not driven by the global layout alone.

Further evidence that contextual cuing may not stem from global configuration alone came from experiments (Chun \& Jiang, 1998) in which only half of the distractor locations were repeated. The presence of novel locations on each repetition produced different configurations from one block to another, yet the subjects still showed a significant, although reduced, contextual cuing effect. This can naturally be explained as the learning of individual targetdistractor associations. But it is also consistent with the possibility that subjects can extract the family resemblance across different variations of the old configuration.

In sum, past studies have not distinguished learning of the emergent configuration formed by all items (global configuration) or some of the items (subsets of the configuration) from learning of each individual target-distractor pair. Although configural learning is considered to be a characteristic of contextual cuing (Chun \& Phelps, 1999), there is no diagnostic evidence as to whether subjects actually form an imaginary configuration or whether they simply learn to associate the target with each individual distractor location. The present study was designed to separate these two types of cues and to examine their roles in contextual cuing. We distinguished between learning to associate the target with each distractor location and learning to associate the target with the pattern formed by the distractors. The latter will be referred to as configural learning or pattern learning, although the pattern or configuration itself may have local subsets with which to cue the target. This distinction will become clear in the context of the experiments, described next.

We tested subjects in two experiments, both involving a training session and a transfer session. In Experiment 1, during the transfer session we preserved the learned individual target-distractor pairs but disrupted the overall configuration formed by all the items or by adjacent items. In Experiment 2, we preserved the learned configuration but disrupted individual distractor locations. If the subjects learned to associate only the distractor configuration with the target, they should show transfer in Experiment 2 but not in Experiment 1. Conversely, if the subjects learned to associate only each distractor location individually with the target, they should show transfer in Experiment 1 but not in Experiment 2.

\section{EXPERIMENT 1 Is Preserved Configuration Necessary?}

In this experiment, we first trained subjects on 36 displays that contained 36 sets of distractor locations and 18 target locations. Each target location was paired with two sets of distractor locations on different trials. For example, one distractor set (e.g., Locations 1-10) was paired with Target Location $\mathrm{X}$ on Trial $n$, and another distractor set (e.g., Locations 11-20) was paired with the same Target Location X on Trial $m$. After 20 blocks of training, the subjects had seen Display 1 (i.e., Target Location X + Distractors 1-10) 20 times and Display 2 (i.e., Target Location X + Distractors 11-20) 20 times. There were 18 different target locations, each paired with two distractor sets. During transfer, the subjects were tested on old displays, new displays, and recombined displays. The old displays were the same as the ones seen during training. The new displays comprised old target locations and new distractor locations. The difference between old and new displays reflects the standard contextual cuing. The re- 
combined condition separated learning of the configuration from learning of individual locations. In the recombined condition, half of the items from one old distractor set were recombined with half of the items from another old distractor set. For example, the subjects now saw Target Location X + Distractors 1-5 + Distractors 11-15. In terms of the pattern or configuration formed by the distractors, because the 11 locations on the recombined displays had never been simultaneously presented before, the configuration had changed from learning to transfer. But every distractor location had been paired with the target location during training, so individual pairwise association was maintained. Thus, if spatial contextual cuing is driven primarily by learning of the configuration, then RTs in the recombined condition should be slower than RTs in the old condition. If, however, the learning of each distractor location is sufficient to drive contextual cuing, then RTs in the recombined condition should be similar to those in the old condition. Figure 1 shows a schematic sample of the design.

Method
Subjects
Fifteen naive subjects, 18-27 years old, volunteered for the ex-
periment. They all had normal or corrected-to-normal visual acuity.
Equipment
The subjects were tested individually in a room with normal light-
ing. They viewed a computer screen from an unrestricted distance of
about $57 \mathrm{~cm}$, at which distance $1 \mathrm{~cm}$ corresponds to $1^{\circ}$ of visual
angle.

\section{Materials}

Each visual search trial contained 11 items (1 target and 10 distractors) presented at randomly selected locations within an invisible $12 \times 8$ matrix $\left(23.4^{\circ} \times 15.6^{\circ}\right)$. Each item contained two line segments and was either an $\mathrm{L}$ or a $\mathrm{T}\left(0.9^{\circ} \times 0.9^{\circ}\right)$. The target, a unique $\mathrm{T}$ presented among rotated Ls, could point either to the left or to the right. The subjects pressed a left key for a leftward $\mathrm{T}$ and a right key for a rightward $\mathrm{T}$. The items were presented in white on a midgray background.

\section{Design}

The experiment was divided into 20 blocks of training (36 trials each) and 3 blocks of transfer ( 36 trials each). Prior to the training blocks, 18 target locations were randomly drawn from the $12 \times 8$ grid. For each target location, 20 distractor locations were randomly chosen and divided into two sets of 10 locations. Each set of 10 was paired with the target location once per block. The 36 trials were then intermixed in presentation, with the constraint that the target location would not be repeated on consecutive trials.

Immediately after training was the transfer test. The trials were divided equally into three conditions: old, recombined, and new (see Figure 1). The old displays were the same as those seen during training. The new displays contained the same 18 target locations, each appearing twice, paired with newly selected distractor locations. The recombined displays contained the 18 target locations, each appearing twice, paired with two different recombinations of the trained displays. Half of the distractors from each of the two trained sets that were initially paired with the target location were recombined to form one display, and the other half from each set were recombined to form another display. The three conditions were randomly intermixed and divided into three blocks.

\section{Trial Sequence}

Each trial started with a fixation point for $800 \mathrm{msec}$, followed by a visual search display that lasted until a response was made. Accu-

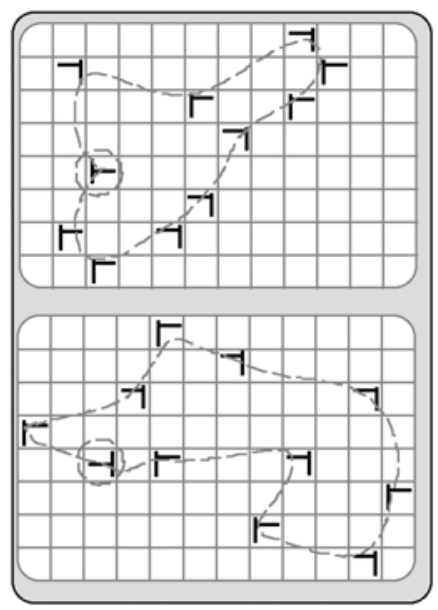

A. Learning + Transfer: Old

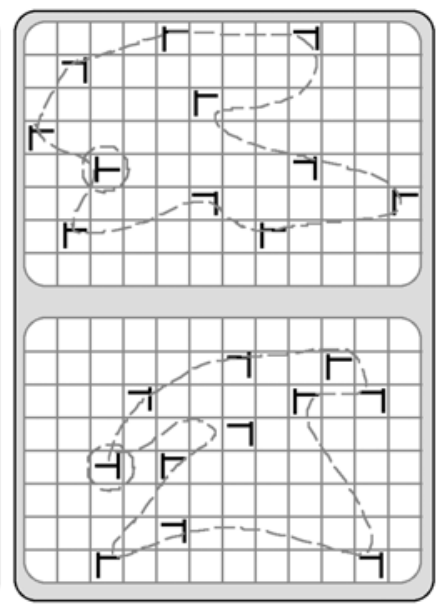

B. Transfer: Recombination

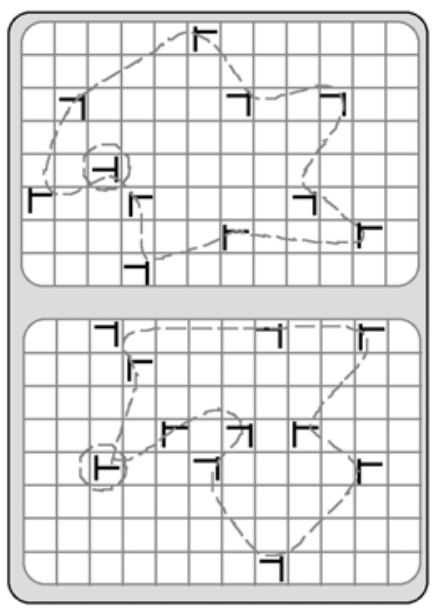

C. Transfer: New distractors

Figure 1. Schematic drawing of the conditions tested in Experiment 1. Panel A shows two distractor sets paired with the same target location on different trials. These displays were seen 20 times during training and once during transfer (the old condition). Panel B shows two recombinations of the trained displays. Half of the distractors were drawn from one trained display and the other half from another trained display (the recombined condition). Panel $\mathrm{C}$ shows new distractor sets paired with the trained target location (the new condition). The target identity was randomly chosen from trial to trial. In the actual experiment, the background grid, the circle marking the target, and the imaginary layout were all invisible. 
racy feedback followed each response. One second later the next trial commenced. The mean RT of a block was displayed during the break between blocks. The subjects were instructed to respond as quickly as possible without sacrificing accuracy. They were not informed of the repetition during training, nor were they informed of the transfer.

\section{Results}

\section{Learning}

Mean accuracy during training ranged from $94 \%-97 \%$ and did not significantly change over time $[F(19,266)=$ $1.45, p>.10]$. We then calculated the median RT for each subject. Figure 2 (left) shows the group mean, separately for each training block. The main effect of block on RT was significant $[F(19,266)=18.66, p<.001]$, showing a significant speed improvement as the experiment progressed. This improvement may reflect procedural learning in visual search (Schneider \& Shiffrin, 1977) as well as specific learning of the repeated displays (Chun \& Jiang, 1998).

\section{Transfer}

During the testing phase, mean accuracy for the old, new, and recombined conditions was $96 \%, 95.5 \%$, and $94.4 \%$, respectively. They were not significantly different from one another $[F(2,28)=1.03, p>.30]$. We then filtered out incorrect trials and calculated the median RT for each subject (mean and median RTs produced the same pattern of results). The group mean is plotted in Figure 2 (right).

Specific learning of the repeated display-contextual cuing - was revealed by a significant difference between the old and the new conditions during transfer $[t(14)=$ $3.20, p<.006]$. The median RT in the old condition was $134 \mathrm{msec}$ faster than that in the new condition. Surprisingly, the median RT in the recombined condition was $122 \mathrm{msec}$ faster than that in the new condition $[t(14)=$ $2.32, p<.036$ ] and only $12 \mathrm{msec}$ slower than that in the old condition. The latter difference was not significant $[t(14)=0.33, p>.50]$. None of the subjects showed a significant difference in RTs between the old and the recombined conditions. On a sign test, eight of the subjects were faster in the old than in the recombined condition, and the other seven showed the reverse pattern. Thus, the data revealed no hint of a reliable difference between the old and the recombined conditions.

\section{Discussion}

In this experiment, we tested whether preserving the learned configuration was necessary for contextual cuing. To our surprise, performance was not affected by a change in configuration. Although the significant advantage of the recombined condition over the new condition was not unexpected, given that repeating half of the distractor locations was sufficient to produce a significant (but reduced) effect (Chun \& Jiang, 1998), the lack of a difference between the old and the recombined conditions was surprising. This finding indicates that the subjects learned to associate the target with individual distractor locations.

This finding is unexpected, given that learning individual distractor locations may be more difficult than learning the global configuration. To represent each location, subjects would have to rely on an environment-based or viewer-based frame of reference. Learning the configuration allows subjects to rely on the relative spatial locations to represent distractors, providing an additional frame of reference. Configural learning also reduces the relevant pieces of information from many (individual locations) to one (configuration). These arguments, however, only elucidate reasons why a display configuration may be learned. They do not preclude the possibility that individual locations can also be learned.

The association between the target and each individual distractor may be acquired because of the serial manner of conjunction search. The global configuration may be less salient once subjects start to search the items one at a time. The distractors visited prior to finding the target become potentially predictive of where the target is. This experi-
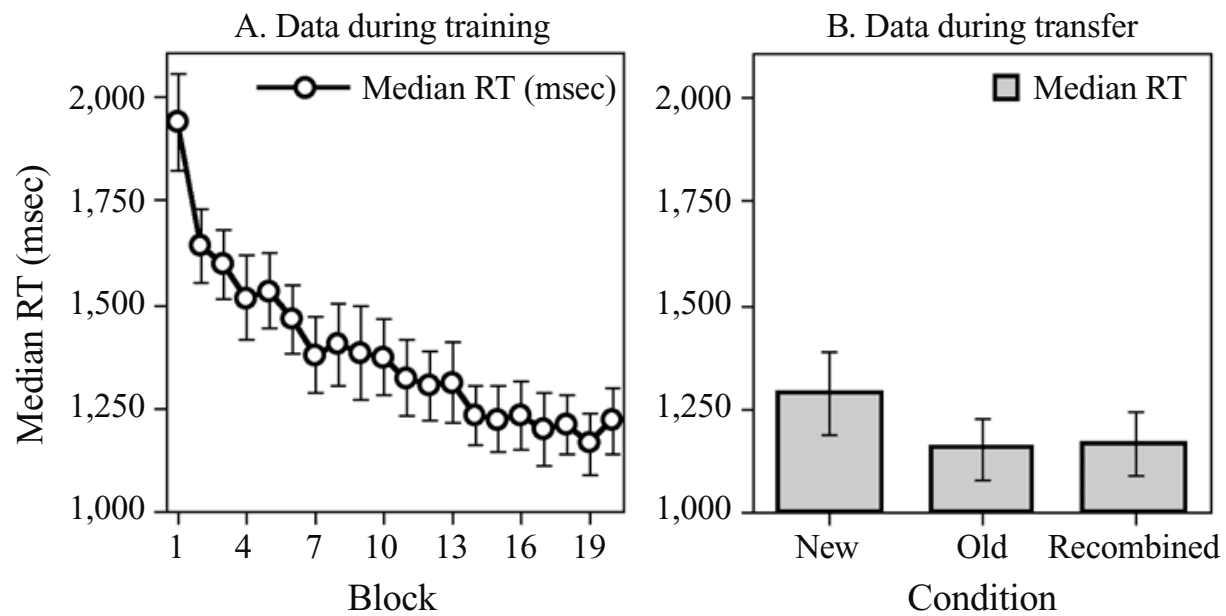

Figure 2. Median response time (RT, in milliseconds) data from Experiment 1. Error bars show standard error of the intersubject variation. 
ment reveals that such associative learning is powerful. Because in normal spatial navigation we rarely have a bird'seye view of the entire environmental layout, pairwise associative learning may be an important skill with which to navigate natural environments.

\section{EXPERIMENT 2 Are Preserved Individual Locations Necessary?}

Experiment 1 showed that learning of the configuration is not necessary to drive contextual cuing. This raises the question about whether or not the pattern formed by all distractors is ever learned. In Experiment 2, we preserved the configuration from learning to transfer but changed individual item locations. If the global configuration is never learned, then there should be no transfer when individual item locations move.

The subjects were first tested in 24 blocks of the training session. Unlike the blocks in Experiment 1, which contained only repeated "old" displays during the training session, in Experiment 2 each block contained a random mixture of old and new displays. ${ }^{1}$ After training, the subjects were tested in displaced and rescaled old displays: displaced $4.5^{\circ}$ to the left, right, up, or down and expanded or contracted by a factor of 1.25 . This linear transformation of the original display did not change the configuration or the relative location among items but did change exactly where each item was with respect to the computer screen and the viewer. If preserving the learned individual distractor locations is necessary for contextual cuing, we should not observe any transfer.

Two groups of subjects were tested in two versions of the experiment. In Experiment 2A, all the items were white so that they would form one perceptual group. In Experiment 2B, half of the distractors were green and the other half were red, and they were segregated into two color groups. The target was randomly chosen to be red or green on each trial. During transfer, some of the green distractors swapped color with some of the red distractors. Baylis and Driver (1992) showed that color similarity could override spatial proximity, so that distant but similarly colored items formed one perceptual group even when color grouping was irrelevant to the search task. Accordingly, because some of the red items swapped color with some of the green items, the perceptual grouping formed by the items changed from learning to transfer. Such changes in color grouping did not affect the global configuration formed by all the items but did disrupt local color grouping. Will learning of an old display transfer to a resized, displaced, and locally regrouped display?

\section{Method}

\section{Subjects}

Twenty subjects were tested in this experiment, half in Experiment $2 \mathrm{~A}$ and half in Experiment 2B.

\section{Materials}

Experiments 2A and 2B differed only in item colors. In Experiment 2A, all the items were white. In Experiment 2B, half of the dis- tractors (five) were red, and half (five) were green; the colors of the items were consistently repeated during the 24 blocks of training. During transfer, two of the previously green distractors swapped color with two of the previously red distractors; the items that changed colors were randomly determined on each trial. The two versions of the experiment were otherwise identical.

Each display contained 1 target and 10 distractors, similar to those in Experiment 1. During training, the items were presented at locations randomly chosen from an invisible $12 \times 8$ matrix that subtended $18^{\circ} \times 12^{\circ}$. The matrix was centered on the monitor (coordinate 0,0 ). During transfer, the items were presented on an invisible $12 \times 8$ matrix that subtended either $22.5^{\circ} \times 15^{\circ}$ or $14.4^{\circ} \times 9.6^{\circ}$. The matrix was presented to the left $(-4.5,0)$, to the right $(4.5,0)$, above $(0,4.5)$, or below $(0,-4.5)$ the monitor's center. Thus, during transfer the old displays were linearly transformed by the following equation:

$$
\text { transfer display }=a * \text { old display }+b,
$$

where $a$ is the rescaling factor ( $a=1.25$ or 0.8 ) and $b$ is the displacement factor $\left(b=4 \cdot 5^{\circ}\right)$.

\section{Design}

There were 24 blocks in the training session (each had 24 trials) and 1 block in the transfer session (192 trials). During training, each block contained 12 new and 12 old displays, each associated with a distinct target location that was repeated across blocks. Distractor locations were repeated only for the old displays. To reduce noise, we matched the eccentricity of the target locations for the new and the old displays. For example, if the target on one old display was $5^{\circ}$ away from fixation in the upper left quadrant, then the target on a new display would be $5^{\circ}$ from fixation in one of the other three quadrants.

Immediately following training, the subjects were tested in the transfer session. There were 96 trials of new displays and 96 trials of transformed old displays. Each of the 12 old displays was transformed in eight ways ( 2 rescaling $\times 4$ displacement). To control perfectly for target locations, we created new displays whose target locations were the same as the target locations of the old displays. The new displays were likewise rescaled and displaced in the same manner as were the old displays. The only difference between the new and the old transformed displays was the distractor configuration. Only for the old displays was the global configuration preserved from training to transfer. For the new displays, distractor locations were randomly chosen on each trial. Figure 3 shows a schematic sample of the conditions. In Experiment 2B, two of the previously red distractors became green, and two of the previously green distractors became red.

The 192 trials were randomly intermixed and divided into eight blocks. The subjects viewed the displays from $60 \mathrm{~cm}$ away from the computer monitor. A chin- and a headrest were used to fix the viewing position. The subjects were not informed of possible repetitions. They underwent training and transfer within a single 45-min session. The other aspects of the experiment were the same as those in Experiment 1.

\section{Results}

In the following paragraphs, we will first report Experiments $2 \mathrm{~A}$ and $2 \mathrm{~B}$ separately and then will pool data across the two versions.

\section{Experiment 2A: Rescaling and Displacing the Learned Configuration}

Mean accuracy ranged from $93 \%$ to $99 \%$ across blocks. It was not affected by condition, block, or the interaction (all $F \mathrm{~s}<1$ ). Correct trials were then entered into RT tests. The mean of the individual subjects' median RTs are shown by solid symbols in Figure 4. 
A. Learned display

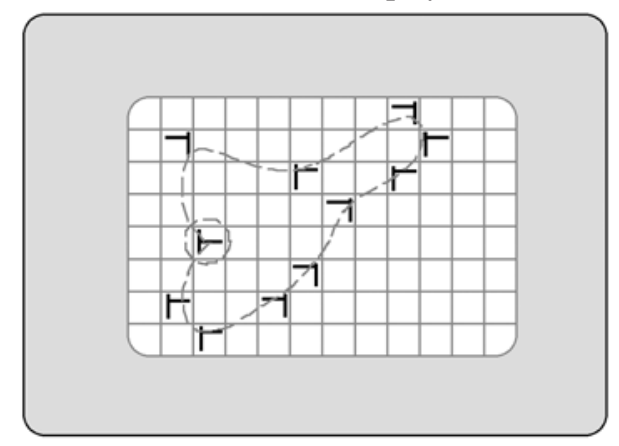

B. Transformed old display

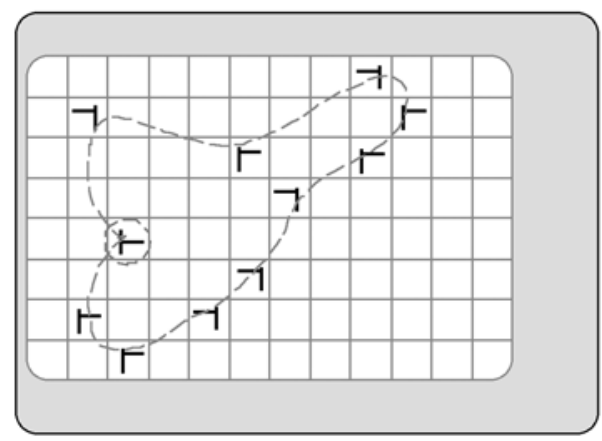

C. Transformed new display

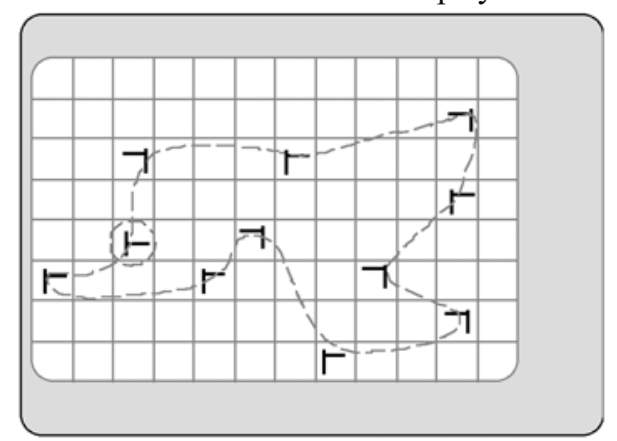

Figure 3. A schematic sample of the displays tested in Experiment 2. Panel A shows a learned display. Panel $B$ is the transformed display in which the learned display was expanded (or contracted) by a factor of 1.25 and displaced $4.5^{\circ}$. Panel $\mathrm{C}$ is a transformed new display. The gray background represents the computer monitor. The subjects viewed the displays at a fixed (by a chinrest) position from $60 \mathrm{~cm}$ away. The background grid, imaginary layout, and target location marker were invisible during the experiment. The items were white in Experiment $2 \mathrm{~A}$. Half of the items were red and the other half were green in Experiment 2B.

Learning. An analysis of variance (ANOVA) on RTs during the learning session, with condition (new vs. old) and block (1-24) as factors, revealed a significant main effect of condition $[F(1,9)=10.90, p<.009]$ and of block $[F(23,207)=7.18, p<.001]$, but no interaction $[F(23,207)=1.01, p>.40]$. The average of the last 192 trials of training (Blocks 17-24) showed a mean RT of $891 \mathrm{msec}$ for the new condition and a mean RT of $811 \mathrm{msec}$ for the old condition. The difference was significant $[t(9)=$ $3.62, p<.006]$. Thus, during the last eight blocks of training, the subjects showed significantly faster RTs to the old displays. But was the learning preserved during transfer?

Transfer. We first pooled data across all 192 trials of transfer to increase statistical power. An analysis on each transfer type will be presented later. The mean of the median RTs for the transformed old displays was $811 \mathrm{msec}$, which was significantly faster than that for the new displays (854 msec) $[t(9)=2.53, p<.032]$. Thus, some learning was preserved even when none of the individual item locations was kept constant from training to transfer in an environment-based or viewer-centered frame of reference. The magnitude of saving was smaller during transfer $(43 \mathrm{msec})$ than during the last eight blocks of training
(80 msec). However, because displays tested during transfer differed from displays tested during learning in size and eccentricity, they were not directly comparable. Statistically, there was no interaction between condition (new vs. old) and epoch (the last eight blocks during learning vs. transfer session) $[F(1,9)=1.51, p>.25]$.

\section{Experiment 2B: Rescaling, Displacing, and Perceptually Regrouping Learned Displays}

Mean accuracy ranged from $94 \%$ to $100 \%$ in different blocks. It was not affected by condition, block, or the interaction in either the learning or the transfer session (all $\left.F_{\mathrm{s}}<1.20, p \mathrm{~s}>.30\right)$.

Learning. The mean of individual subjects' median RTs (correct trials only) revealed a significant main effect of condition, with faster RTs in the old than in the new condition $[F(1,9)=13.65, p<.005]$, and a significant main effect of block $[F(23,207)=10.21, p<.0001]$, but no interaction. By pooling data across the last eight blocks of training, we observed significantly faster RTs in the old than in the new condition (mean difference, $120 \mathrm{msec}$ ) $[t(9)=3.59, p<.006]$. Thus, after 24 blocks of training, the subjects had learned the old, repeated displays. 


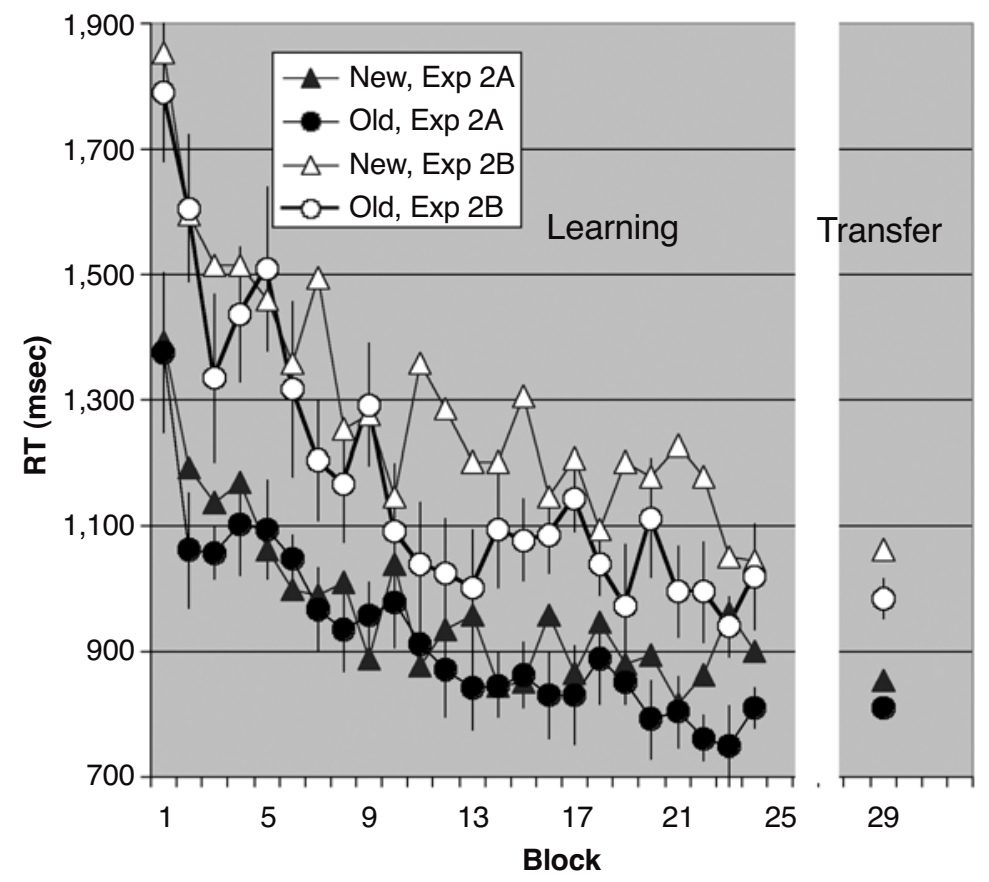

Figure 4. Median response time (RT, in milliseconds) data from Experiment 2. Error bars show standard error of the difference between the new and old conditions. The data obtained during transfer were averaged together to produce one value for the new and another for the old condition.

Transfer. The rescaled, displaced, and perceptually regrouped old displays were still responded to more quickly than were the new displays ( $M=984 \mathrm{msec}$ for the transformed old condition; $M=1,062 \mathrm{msec}$ for the new condition; difference $=78 \mathrm{msec})[t(9)=2.41, p<.04]$. This finding replicated that in Experiment $2 \mathrm{~A}$ in that the change in the absolute individual locations could not remove the learning effect. It further shows that learning persisted despite a change in local color grouping.

\section{Combined Analysis: Experiments 2A and 2B}

Because the two versions of the experiment produced similar patterns of results, we pooled data across the two experiments to increase statistical power, with display color as a between-subjects factor.

Learning. In addition to the significant main effects of block (general procedural learning) and condition (specific learning of the old displays), there was a significant main effect of subject group $[F(1,18)=8.00, p<.011]$ and a marginally significant interaction between condition and group $[F(1,18)=3.77, p<.07]$. The subjects in Experiment $2 \mathrm{~B}$ were slower than were the subjects in Experiment $2 \mathrm{~A}$, and they showed a marginally larger contextual cuing effect. This is consistent with previous observations (Chun \& Jiang, 1998) that contextual cuing is larger when items have heterogeneous colors than when they have homogeneous ones, suggesting that the color manipulation was a noticeable factor.
Transfer. Here, too, the subjects in Experiment 2B showed slower RTs than did the subjects in Experiment 2A $[F(1,18)=4.52, p<.05]$. RTs were significantly faster in the transferred condition than in the new condition $[F(1,18)=10.96, p<.004]$. The interaction between experiment and condition was not significant $[F(1,18)<1$, n.s.].

Because the factor of experimental group did not significantly interact with any of the following factors $(F \mathbf{s}<1)$, we combined data from all 20 subjects to examine how the transfer effect was affected by the kind of change. We entered condition (new vs. old), size (contraction vs. expansion), and displacement direction (up, down, left, or right) in a repeated measures ANOVA. The main effect of condition was significant $[F(1,19)=19.04, p<.0001]$, but none of the other effects were (all $p \mathrm{~s}>.12$ ). RT was not influenced by whether the display became smaller or larger $[F(1,19)=1.22, p>.25]$ or by which direction it shifted to $[F(3,57)=2.01, p>.12]$, nor did any of these effects interact with condition $\left(F_{\mathrm{S}}<1\right)$.

Table 1 shows mean RTs separately for each condition (new vs. old) and each transfer type. Pairwise $t$ tests revealed statistically significant transfer effects when an old display expanded and shifted to the left or to the right and when an old display contracted and shifted up or down. The other conditions showed a smaller (but positive) transfer effect. Although the transfer effects were not equivalent in magnitude across the eight transfer types, the dif- 
Table 1

Group Mean of Individuals' Median RTs (in Milliseconds) During the Transfer Session in Experiment 2, Pooled Across Experiments 2A and 2B

\begin{tabular}{lccrrrr}
\hline $\begin{array}{c}\text { Displacement } \\
\text { Direction }\end{array}$ & New & Old & Difference & $S E$ & $t(19)$ & $p$ \\
\hline \multicolumn{7}{c}{ Contract } \\
Down & 1,028 & 1,008 & 20 & 38 & 0.51 & .612 \\
Left & 1,059 & 924 & 136 & 63 & 2.14 & .046 \\
Right & 1,038 & 936 & 101 & 49 & 2.06 & .053 \\
Up & 1,072 & 1,028 & 44 & 46 & 0.95 & .352 \\
& 1,033 & 915 & 118 & 50 & 2.37 & .029 \\
Down & 1,015 & 972 & 43 & 41 & 1.06 & .302 \\
Left & 970 & 959 & 11 & 50 & 0.22 & .827 \\
Right & 1,094 & 949 & 145 & 57 & 2.53 & .020 \\
Up & \multicolumn{7}{c}{ Expand } \\
\hline
\end{tabular}

ference was not significant in the ANOVA test (see the previous paragraph).

Finally, to examine whether the cuing effect shown in the transfer session was smaller than the initial learning effect during the last eight blocks, we performed an ANOVA on session (the last block of learning vs. the transfer session) and condition. Here, we observed a significant main effect of condition $[F(1,19)=7.19, p<.015]$ but no effect of session nor an interaction $\left(F_{\mathrm{S}}<1\right)$. Thus, the overall RT had already reached asymptote and did not further improve during the transfer session, and the effect of learning the old displays showed a statistically complete transfer.

\section{Discussion}

By rescaling and displacing the learned displays, we changed the locations occupied by each item on the display with respect to the computer screen and to the viewer. However, the relative spatial relation among the items was maintained. The results showed a significant transfer effect, suggesting that preserving exactly where each distractor was on the display was unnecessary for contextual cuing.

We note that when the relative spatial relation among items is kept constant, a transfer effect can be observed not only when subjects learn the overall configuration formed by all items, but also when they learn local subsets of the global configuration. ${ }^{2}$ These two aspects of the emergent shape formed by the items - global pattern and local subsets - are almost always correlated with each other. In Experiment 2B, by introducing a change in local color grouping, we attempted to dissociate the two effects. If color grouping had registered and subsets had been formed for similarly colored items (Baylis \& Driver, 1992), then a change in color grouping would have disrupted these local subsets but not the global configuration. The nearly complete transfer effect across perceptually regrouped displays suggests that the global configuration itself may be sufficient to drive contextual cuing. However, subjects could have ignored the color segregation altogether and might have relied on spatial proximity rather than color similarity to form local subsets of the configuration, in which case color change would not actually produce a change in perceptual grouping. Thus, the results from Experiment 2B are open to alternative interpretations: Either global configuration alone was sufficient to drive contextual cuing in such a way that the change in local subsets was not disruptive or else the color grouping did not influence how local subsets were segregated.

The separation between the global configuration and local subsets is difficult, not only because they are highly correlated but also because, unlike actual objects, the formation of a configuration on the basis of discrete locations is ambiguous. Whether one sees the identical star constellation as the Great Bear or the Big Dipper depends on how the pattern formed by the stars is perceived and how the same constellation is parsed into subsets. Because of the difficulty in assigning a uniform global pattern to such displays and in segregating it into parts, it will not be easy to separate the two effects experimentally. One could obviate this problem by actually assigning an outline shape to the discrete locations, just as the outline of a bear is overlaid on the star constellation. Thus, for example, one might draw the same outline shape and move the locations to different parts of the outline, or one might draw two different outlines through the same individual locations. This would be an alternative method to dissociate the effects of absolute distractor locations from the global configuration formed by the locations, although here, too, the global shape and the local subsets would still be correlated. The problem with this manipulation, however, is the assumption that subjects would pay attention to the outline shape and would move attention along the contour of the shape. If subjects choose to ignore the outline and still search through the items in a semi-random order, the outline shape would not have an effect. Even if subjects were to rely on the outline shape, they might not do so under conditions in which it is absent. ${ }^{3}$

However, a less contentious division can be drawn between the absolute spatial locations of each individual location and the relative spatial relationships among the locations. The former is based on an environment-centered or viewer-centered frame of reference, whereas the latter is based on an object-centered frame of reference. Although we cannot easily make finer distinctions between the global configuration and local subsets, it is relatively straightforward to distinguish between two ways of representing the distractors - individually or relationally. The two experiments reported in this article indicate that both may be represented during visual search and that preserving either cue is sufficient to lead to a nearly complete contextual cuing effect.

\section{GENERAL DISCUSSION}

Together, the two experiments in the present study provide evidence for two types of learning in contextual cuing. Subjects learned not only the association between the dis- 
tractor pattern (or subsets of the pattern) and the target location, but also the association between each individual distractor location and the target location. Disrupting either the relative spatial relation among the items (Experiment 1) or the exact spatial locations of individual items on the display (Experiment 2) did not remove contextual cuing, as long as the other cue was present. The presence of local target-distractor associations alone appeared sufficient to drive full contextual cuing (Experiment 1). The presence of global configural association alone also appeared to produce a cuing effect that was statistically indistinguishable from original learning (Experiment 2). Implicit learning of such visual displays can thus occur at different hierarchical levels. Learning at one level survives the loss of information at the other level, suggesting that contextual cuing is a robust form of visual learning.

Both forms of learning are important for spatial navigation because subjects rarely have a bird's eye view of a global spatial layout. Instead, they search the visual environment serially, and the associative learning between individual distractor locations and the target would be useful under such conditions. However, because global configuration allows items to be represented in an object-centered frame of reference and reduces the pieces of important information from many (locations) to one (layout), it is also a useful cue.

Although this study does not directly address the issue of relative importance between configural learning and individual location learning, we conjecture that the configural learning may be more important as subjects acquire higher levels of proficiency within a certain environment. After repeated navigation within the same environment, the subjects may gradually integrate discrete, isolated locations into one spatial schema. They might then rely more on the relative distractor locations than on isolated distractor-target associations to locate the target. In addition, tasks that require or permit the allocation of attention to all the items in parallel, such as change detection or feature search, may also increase the reliance on learning the global layout. Neither change detection nor feature search was tested in this study, however, because additional studies need to first establish that such tasks can be enhanced by the repetition of the distractor locations. Future studies using these paradigms may allow us to determine when the global layout is preferred over individual locations when attention is allocated to all the items in parallel.

Additional studies should also address whether there is a limit on the learning of individual distractor-target pairs. Increasing the number of distractors on a given display may weaken the associative strength between each distractortarget pair. In addition, as the same distractor location is associated with an increasing number of possible target locations, its predictive value will decrease. Finally, although recombining two learned old displays still results in robust contextual cuing, recombining 5 or 10 learned old displays may produce a much smaller effect. Nonetheless, although there may be many conditions under which individual pairwise learning will be reduced in strength, we have established in the present study that contextual cuing relies significantly on individual associations as well as on configural learning.

\section{REFERENCES}

Ballard, D. H., Hayhoe, M. M., \& Pelz, J. B. (1995). Memory representations in natural tasks. Journal of Cognitive Neuroscience, $\mathbf{7}_{2}$ 66-80.

BAYLIS, G. C., \& Driver, J. (1992). Visual parsing and response competition: The effect of grouping factors. Perception \& Psychophysics, 51, 145-162.

CHUN, M. M. (2000). Contextual cuing of visual attention. Trends in Cognitive Sciences, 4, 170-178.

ChUn, M. M., \& JiAnG, Y. (1998). Contextual cueing: Implicit learning and memory of visual context guides spatial attention. Cognitive Psychology, 36, 28-71.

CHUN, M. M., \& JIANG, Y. (1999). Top-down attentional guidance based on implicit learning of visual covariation. Psychological Science, $\mathbf{1 0}$, 360-365.

CHUN, M. M., \& JiANG, Y. (2003). Implicit, long-term spatial contextual memory. Journal of Experimental Psychology: Learning, Memory, \& Cognition, 29, 224-234.

Chun, M. M., \& Phelps, E. A. (1999). Memory deficits for implicit contextual information in amnesic subjects with hippocampal damage. Nature Neuroscience, 2, 844-847.

HAYноE, M. (2000). Visual routines: A functional account of vision. $\mathrm{Vi}$ sual Cognition, 7, 673-704.

IRWIN, D. E. (1992). Perceiving an integrated visual world. In D. E. Meyer \& S. Kornblum (Eds.), Attention \& performance XIV: Synergies in experimental psychology, artificial intelligence, and cognitive neuroscience (pp. 121-142). Cambridge, MA: MIT Press.

JiAng, Y., \& ChUn, M. M. (2001). Selective attention modulates implicit learning. Quarterly Journal of Experimental Psychology, 54A, 1105-1124.

JiAnG, Y., Olson, I. R., \& ChUn, M. M. (2000). Organization of visual short-term memory. Journal of Experimental Psychology: Learning, Memory, \& Cognition, 26, 683-702.

KeELE, S. W., HAWKINS, H. L. (1982). Explorations of individual differences relevant to high level skills. Journal of Motor Behavior, $\mathbf{1 4}$, 3-23.

LEVIN, D. T., \& Simons, D. J. (1997). Failure to detect changes to attended objects in motion pictures. Psychonomic Bulletin \& Review, $\underline{4}_{2}$ 501-506.

LiU, J., HARris, A., \& KANWISHER, N. (2002). Stages of processing in face perception: An MEG study. Nature Neuroscience, 5, 910-916.

MACK, A., \& Rock, I. (1998). Inattentional blindness. Cambridge, MA: MIT Press.

Maljkovic, V., \& Nakayama, K. (1994). Priming of popout: I. Role of features. Memory \& Cognition, 22, 657-672.

Maljkovic, V., \& Nakayama, K. (1996). Priming of popout: II. The role of position. Perception \& Psychophysics, 58, 977-991.

Nissen, M. J., \& Bullemer, P. (1987). Attentional requirements of learning: Evidence from performance measures. Cognitive Psychology, 19, 1-32.

OLSON, I. R., \& Chun, M. M. (2001). Temporal contextual cuing of visual attention. Journal of Experimental Psychology: Learning, Memory, \& Cognition, 27, 1299-1313.

OLSON, I. R., \& CHUN, M. M. (2002). Perceptual constraints on implicit learning of spatial context. Visual Cognition, 9, 273-302.

O’REgan, J. K. (1992). Solving the "real" mysteries of visual perception: The world as an outside memory. Canadian Journal of Psychology, 46, 461-488.

PASHLER, H. (1994). Dual-task interference in simple tasks: Data and theory. Psychological Bulletin, 116, 220-244.

PASHler, H. (1998). The psychology of attention. Cambridge, MA: MIT Press.

PotTER, M. C. (1976). Short-term conceptual memory for pictures. 
Journal of Experimental Psychology: Human Learning \& Memory, 29, 637-650.

REBER, A. S. (1989). Implicit learning and tacit knowledge. Journal of Experimental Psychology: General, 118, 219-235.

Rensink, R. A., O'Regan, J. K., \& ClarK, J. J. (1997). To see or not to see: The need for attention to perceive changes in scenes. Psychological Science, 8, 368-373.

SCHNEIDER, W., \& SHIFFRIN, R. M. (1977). Controlled and automatic human information processing: I. Detection, search and attention. Psychological Review, 84, 1-66.

SHORE, D. I., \& KLEIN, R. M. (2000). The effects of scene inversion on change blindness. Journal of General Psychology, 127, 27-43.

Thorpe, S., FiXe, D., \& MARLOT, C. (1996). Speed of processing in the human visual system. Nature, 381, 520-522.

Wolfe, J. M., KLEMPEN, N., \& DAHLEN, K. (2000). Postattentive vision. Journal of Experimental Psychology: Human Perception \& Performance, 26, 693-716.

YANTIS, S. (1992). Multielement visual tracking: Attention and perceptual organization. Cognitive Psychology, 24, 295-340.

YAntis, S. (1998). Control of visual attention. In H. Pashler (Ed.), Attention (pp. 223-256). London: Psychology Press.

\section{NOTES}

1. We were constrained by the total number of trials that we could fit in within a 1-h session (approximately 800 trials). Thus, the new condition was not tested in Experiment 1 because each block already contained 36 trials.

2. We thank Jennifer Stolz and an anonymous reviewer for raising this possibility.

3. We tested 15 new subjects in a variation of Experiment 1 in a direct attempt to make the configuration more salient by connecting the imaginary layout of each display. The recombined condition, which maintained individual locations but varied the global configuration, fell halfway between the new and the old condition and was found to be not significantly different from either the new or the old condition, even though the new and the old conditions differed significantly from each other. Thus, configural learning may be stronger when the layout is made more salient, although it might not override the learning of individual locations completely.

(Manuscript received February 25, 2003; revision accepted for publication August 25, 2003.) 